\title{
On a recent reciprocity formula for Dedekind sums
}

\author{
Kurt Girstmair
}

\begin{abstract}
Let $s(a, b)$ denote the classical Dedekind sum and $S(a, b)=12 s(a, b)$. Recently, $\mathrm{Du}$ and Zhang proved the following reciprocity formula. If $a$ and $b$ are odd natural numbers, $(a, b)=1$, then

$$
S\left(2 a^{*}, b\right)+S\left(2 b^{*}, a\right)=\frac{a^{2}+b^{2}+4}{2 a b}-3,
$$

where $a a^{*} \equiv 1 \bmod b$ and $b b^{*} \equiv 1 \bmod a$. In this paper we show that this formula is a special case of a series of similar reciprocity formulas. Whereas Du and Zhang worked with the connection of Dedekind sums and values of $L$-series, our main tool is the three-term relation for Dedekind sums.
\end{abstract}

\section{Introduction and Result}

Let $a$ be an integer, $b$ a natural number, and $(a, b)=1$. The classical Dedekind sum $s(a, b)$ is defined by

$$
s(a, b)=\sum_{k=1}^{b}((k / b))((a k / b)) .
$$

Here

$$
((x))= \begin{cases}x-\lfloor x\rfloor-1 / 2 & \text { if } x \in \mathbb{R} \backslash \mathbb{Z} ; \\ 0 & \text { if } x \in \mathbb{Z}\end{cases}
$$

(see [6, p. 1]). It is often more convenient to work with

$$
S(a, b)=12 s(a, b)
$$

instead. We call $S(a, b)$ a normalized Dedekind sum.

Probably the most important elementary result concerning Dedekind sums is reciprocity law. If $a$ and $b$ are coprime natural numbers, then

$$
S(a, b)+S(b, a)=\frac{a^{2}+b^{2}+1}{a b}-3 .
$$

Recently, Du and Zhang have found the following hitherto unknown reciprocity law (see [2]). If $a$ and $b$ are coprime odd natural numbers, then

$$
S\left(2 a^{*}, b\right)+S\left(2 b^{*}, a\right)=\frac{a^{2}+b^{2}+4}{2 a b}-3,
$$


where $a a^{*} \equiv 1 \bmod b$ and $b b^{*} \equiv 1 \bmod a$.

The proof given in [2] is based on the connection of Dedekind sums and values of $L$-series. The authors of the said paper ask for an elementary proof of their result. Here we give such an elementary proof based on the tree-term-relation of Dedekind sums. Moreover, we show that (2) is a special case of a series of similar reciprocity formulas. Indeed, we have the following.

Theorem 1 Let $a$ and $b$ be coprime natural numbers and $t$ a natural number such that $a^{2}+1 \equiv 0 \bmod t$. Further, let $(b, t)=1$. Then

$$
S\left(t a^{*}, b\right)+S\left(t b^{*}, a\right)=\frac{a^{2}+b^{2}+t^{2}}{t a b}-3+S(a b, t) .
$$

As to the case $t=1$, we note

$$
S\left(a^{*}, b\right)=S(a, b)
$$

(see [6, p. 26]) and $S(a b, 1)=0$. In the case $t=2, a$ and $b$ are odd and $S(a b, 2)=0$. Hence we obtain the following.

Corollary 1 The formulas (11) and (2) are immediate consequences of Theorem 1 in the cases $t=1$ and $t=2$.

Corollary 2 Suppose, in the setting of Theorem 1 , that $b \equiv \pm 1 \bmod t$. Then

$$
S\left(t a^{*}, b\right)+S\left(t b^{*}, a\right)=\frac{a^{2}+b^{2}+t^{2}}{t a b}-3 .
$$

Suppose, on the other hand, that $b \equiv \pm a \bmod t$. Then

$$
S\left(t a^{*}, b\right)+S\left(t b^{*}, a\right)=\frac{a^{2}+b^{2}+t^{2}}{t a b}+ \begin{cases}-t-2 / t, & \text { if } b \equiv a \bmod t \\ t+2 / t-6, & \text { if } b \equiv-a \bmod t .\end{cases}
$$

As to (5), note that $(a b)^{2} \equiv-1 \bmod t$, which shows that $S(a b, t)=0$ (see [6, p. 28]). In the case of (6) ), we use $S(1, t)=t+2 / t-3$ (which is an immediate consequence of (10) and $S(-a, b)=-S(a, b)$ (see [6, p. 26]).

Remark. The natural numbers $t$ such that there is a natural number $a$ with $a^{2}+1 \equiv 0$ mod $t$ can be characterized as follows: $t=m$ or $t=2 m$, where $m$ is a natural number whose prime divisors are all $\equiv 1 \bmod 4$ (this includes $m=1$ ).

Example. Let $t=5, a$ such that $a^{2}+1 \equiv 0 \bmod 5$, and $(a, b)=(b, 5)=1$. This implies $a \equiv \pm 2 \bmod 5$. If $b \equiv \pm 1 \bmod 5$, then

$$
S\left(5 a^{*}, b\right)+S\left(5 b^{*}, a\right)=\frac{a^{2}+b^{2}+25}{5 a b}-3 .
$$

In the remaining case, we have $b \equiv \pm a \bmod 5$. If $b \equiv a \bmod 5$, then (6) reads

$$
S\left(5 a^{*}, b\right)+S\left(5 b^{*}, a\right)=\frac{a^{2}+b^{2}+25}{5 a b}-27 / 5 .
$$

If $b \equiv-a \bmod 5$, we have

$$
S\left(5 a^{*}, b\right)+S\left(5 b^{*}, a\right)=\frac{a^{2}+b^{2}+25}{5 a b}-3 / 5 .
$$




\section{Proof of Theorem 1}

Let $a, b, t$ be natural numbers, $(a, b)=(b, t)=1$, such that $a^{2}+1 \equiv 0 \bmod t$. Put $c=b\left(a^{2}+1\right) / t$. Obviously, $(a, c)=1$. Then [4, Th.4] says

$$
S(a, c)=\frac{\left(b^{2}-1\right) a}{t b}-S(a b, t)+S\left(a t^{*}, b\right) .
$$

where $t t^{*} \equiv 1 \bmod b$. By the reciprocity law (1),

$$
S(a, c)=-S(c, a)+\frac{a^{2}+c^{2}+1}{a c}-3 .
$$

However, $c \equiv b t^{*} \bmod a$, with $t t^{*} \equiv 1 \bmod a$. Hence $S(c, a)=S\left(b t^{*}, a\right)$. We replace $a t^{*}$ by $t a^{*}$ and $b t^{*}$ by $t b^{*}$ in the respective normalized Dedekind sums (see (44)). Then a short calculation proves Theorem 1 .

We still have to make clear that this remarkably simple proof is based on elementary results. Indeed, (7) follows from the three-term relation

$$
S(a, b)=S(c, d)+\varepsilon S(r,|q|)+\frac{b^{2}+d^{2}+q^{2}}{b d q}-3 \varepsilon
$$

(see [4]). Here $b, d$, are natural numbers, $a, c$ integers, $(a, b)=(c, d)=1, a / b \neq c / d$. Further, $q=a d-b c$ and $\varepsilon$ is the sign of $q$. Finally, $r=a j-b k$, where $j, k$ are integers such that $-c j+d k=1$. The three-term relation, in turn, can be deduced from the composition rule of the logarithm of Dedekind's $\eta$-function (see [1, 3]). An elementary proof of this composition rule is given in [5, §4].

\section{References}

[1] U. Dieter, Beziehungen zwischen Dedekindschen Summen, Abh. Math. Sem. Univ. Hamburg 21 (1957), 109-125.

[2] X. Du, L. Zhang, On the Dedekind sums and its new reciprocity formula, Miskolc Math. Notes 19 (2018), 235-239.

[3] K. Girstmair, Dedekind sums with predictable signs, Acta Arith. 83 (1998), 283-292.

[4] K. Girstmair, On the values of Dedekind sums, J. Number Th. 178 (2017), 11-18.

[5] H. Rademacher, Zur Theorie der Modulfunktionen, J. reine angew. Math. 167 (1931), $312-336$.

[6] H. Rademacher, E. Grosswald, Dedekind sums, Mathematical Association of America, 1972.

Kurt Girstmair

Institut für Mathematik

Universität Innsbruck

Technikerstr. 13/7

A-6020 Innsbruck, Austria

Kurt.Girstmair@uibk.ac.at 